\title{
Application of Matlab Notebook in the Course Teaching of Signal and System
}

\author{
YaXun Zhou, Jianfeng Dong, Xingbin Zeng, Gangyi Jiang \\ College of Information Science and Engineering, Ningbo University \\ Ningbo, China \\ zhouyaxun@nbu.edu.cn
}

\begin{abstract}
Signal and System is a course with a lot of abstracting theories and mathematical derivations, and it is often needed to display the complex waveforms of some signals and simulate the signal processing process in order to better understand the relevant knowledge in the course teaching. To this end, Matlab notebook, which seamlessly links the powerful data-processing and visualization software Matlab with the powerful word-processing software Word, can be adopted in the course teaching and thus the teaching content combined with text and figure or static and dynamic presentation can be realized through it. Also, using Matlab notebook can overcome the cumbersome processes in the traditional multimedia teaching, in which the switch from one application program (for example, the Word) to another one (for example, the Matlab) and vice versa are needed in order to display the signal waveforms or present processing data. This will be beneficial to improve the course teaching progress and effectiveness, and at the same time make up for the some lacks of course experiment to a certain extent.
\end{abstract}

Keywords-signal and system; matlab notebook; course teaching

\section{INTRODUCTION}

Signal and System is an emerging discipline developed rapidly with the computer technology, and it has been now widely applied in various fields of radar, communications, sonar, remote sensing, bio-engineering and so on. Meanwhile, its theories are also the foundations of the Communication Principle, Digital Signal Processing and other professional courses. Therefore, Signal and System is listed as an absolutely required course for many majors such as electronic, information science and communication engineering in the colleges and universities. However, due to its abundant theories and practices of this discipline, Signal and System course is often fallen into such a situation of difficult to teach but more difficult to learn, mainly because that $[1,2]$, on the one hand, it is a discipline based on the High Mathematics, Circuit Theory and other courses with many formulas, mathematical derivation, profound theoretical knowledge and abstracting concepts. The other hand, the Signal and System itself has a large number of complex algorithms and computation. Thus, the students are difficult to visualize the results and in turn have a thorough understanding of the course knowledge if without the computer simulation. Recently, the multimedia teaching methods are introduced in the course teaching of Signal and System. Compared with the traditional blackboard teaching, the multimedia teaching can change the abstracting preaching into the image demonstration and thus greatly stimulate students learning interest. However, there exists some limitations in the traditional multimedia teaching method, for example, it is often needed to switch from the current window to the other application program window such as Matlab window in order to program, execute and then copy the running results to the courseware if one wants to real-time demonstrate or display the visualization of an algorithm. Obviously, this process is more cumbersome and will inevitably affect the course teaching progress and teaching effectiveness.

From the version 5.0 of Matlab software, the MathWorks has developed the Notebook tool which seamlessly links the powerful Microsoft word processing software Word with the powerful data processing and visualization software Matlab together [3-7]. The Notebook tool provides a perfect unity working environment for word processing, scientific computing and engineering design. It not only keeps the full functions of Word software, but also allows the user to directly deliver the Matlab command created in the word document to the Matlab software and execute it, then sends the calculating results and graphics back to the word document, and finally achieves real-time programming, realtime execution and display results in the word document. This is very useful in the multi-media course teaching, in which it is needed to present the corresponding calculation data, display the graphic curves, or needed to change signal parameter several times to demonstrate when teaching the relevant knowledge. Therefore, the Matlab notebook tool is introduced in the course teaching of Signal and System, and practice shows its good teaching effectiveness.

\section{LINKS OF MATLAB WITH WORD}

In order to use powerful Matlab in the Word environment, it is needed to link the two together, which must install Matlab Notebook first.

\section{A. Installation of Matlab Notebook}

It is simple to install Matlab Notebook if the computer has installed Matlab and Word software, one can start Matlab and then input the following statement in its command window:

notebook -setup $\downarrow$

According to the prompt appeared in the screen, select the current version of Word used by the computer to complete the installation of Matlab Notebook. The detailed installation process can be found in some specialized books [3]. 


\section{B. Start of Matlab Notebook}

After installing Notebook successfully, Matlab creates automatically a template named M-book.dot for Word. Mbook template provides user an integrated Word environment to use Matlab and the created word document is usually called as the M-book document. The start of Matlab Notebook is in fact to create a new or open an existed Mbook documents, this can be directly realized in the Matlab command window by executing the following command:

notebook or notebook M-book name $\downarrow$

Or, it is can be done according to the following operation: select "New" command in "File" menu in the Word window first, then select M-book template in the pop-up dialog box. Open an existed M-book document is similar to open a general Word document. The opened M-book document interface is similar to the conventional Word interface, only adds a menu item called the Notebook in the menu bar as shown in Figure 1, and this menu contains a series of Notebook operating commands.

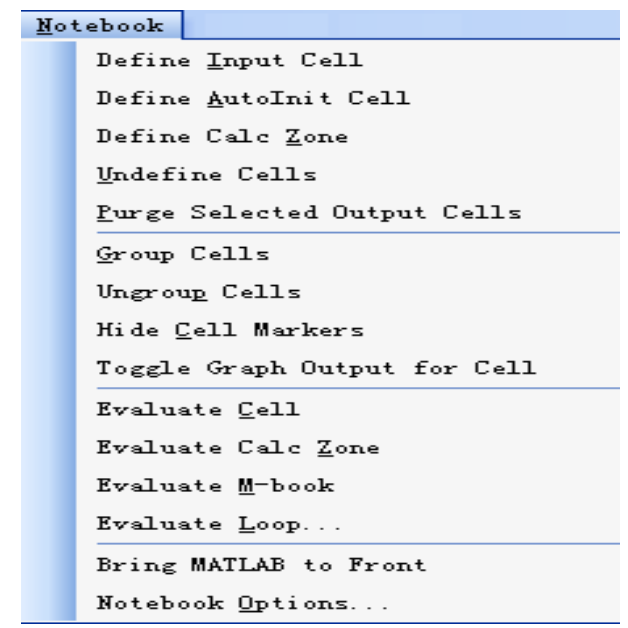

Figure 1. The Notebook menu in the M-book document

\section{Use of Matlab Notebook}

Notebook makes the Word software interact with the Matlab software through the dynamic link library, and the interacted basic unit is the cell. The command sent to the Matlab by M-book document is called the input cell, and any valid Matlab commands created in the document can be defined as the input cell. The input cell sent to the Matlab environment can be executed immediately, and the executed results are stored in the Matlab workspace and at the same time are sent back to the M-book document as the output cell. The main operation of Matlab notebook is to define and then execute the input cell: select the required command first, and then select the "Define Input Cell" command in the Notebook menu or directly press the combined key of Alt $+\mathrm{D}$, thus the selected Matlab commands become as the input cell. Select the "Evaluate Cell" command in the Notebook menu or directly press the combined key of Ctrl+Enter, the input cell can be executed and return the operating results.

\section{EXAMPLE OF APPLICATION}

The course content of Signal and System can be summarized into several important knowledge points [1], and they are the time-domain analysis of linear timeinvariant (LTI) system, Fourier analysis of continuous /discrete time signal and system, sampling and modulation/demodulation of continuous time signal, Laplace transform and continuous time system, and z-transform and discrete time system, respectively. These knowledge points contain many abstracting concepts, esoteric theories, tedious mathematical derivation and the complex algorithms. In order to make the students easy to understand the course knowledge, help them to master the basic concepts, basic principles and analytical methods of signal and system, and meanwhile arouse students learning interest, the teaching contents are made as electronic handouts with the M-book document, in which one can program, run and display in real-time while teaching the relevant knowledge points.

For example, when one teaches the content of continuous time signal sampling of Signal and System, which relates to the Nyquist sampling theorem: to recover the original signal without any distortion from the sampled sequence, the sampling frequency must be greater than the twice maximum frequency of the original signal. Nyquist sampling theorem is a learning difficult point for many students, and only analyzing and comparing the difference of Fourier spectra of discrete-time sequence generated by sampling the continuous-time signal with different sampling frequency, can the students better understand the physical meanings included in the sampling theorem. Now, select continuoustime signal $x_{\mathrm{a}}(t)=\mathrm{e}^{-1000|t|}$ as an example, its highest frequency component is about $2000 \mathrm{~Hz}$ under accuracy of $10^{-5}$.

Start the Notebook and write the following program in the M-book document:

$T=0.00005 ; t=-0.005: T: 0.005$;

$x_{\mathrm{a}}=\exp (-1000 * \operatorname{abs}(t)) ; \%$ continuous-time signal

$T_{\mathrm{s}}=0.0002 ; n=-25: 1: 25$

$x_{1}=\exp \left(-1000 * \operatorname{abs}\left(n * T_{\mathrm{s}}\right)\right)$; \% discrete-time sequence

$K=500 ; k=0: 1: 3^{*} K$; \% Fourier analysis of sequence

$w=\mathrm{pi}^{*} \mathrm{k} / K$;

$X=x_{1} * \exp \left(-\mathrm{j}^{*} n^{\prime *} w\right)$

$X=\operatorname{real}(X)$;

$w=[-f l i p l r(w), w(2: 3 * K+1)]$

$X=\left[\right.$ fliplr $\left.(X), X\left(2: 3^{*} K+1\right)\right]$;

subplot(2,1,1); $\operatorname{plot}\left(t^{*} 1000, X_{\mathrm{a}}, \mathrm{r}^{\prime}\right)$;

xlabel('t /ms'); ylabel(' $x_{1}(t)$ ');

title('Discrete time sequence'); hold on;

stem $\left(n * T s * 1000, x_{1}\right)$; hold off;

subplot(2,1,2); plot(w/pi, $X$, 'b');

xlabel('Frequency in pi units'); ylabel(' $X_{1}(\mathrm{w})$ ');

title('Discrete time Fourier Transform $\left(T_{\mathrm{s}}=0.2 \mathrm{~ms}\right)$ ');

Select the above program first and press the combined key of Alt+D to set it as the input cell, and then press the combined key of Ctrl+Enter, the executing results as shown in Figure 2(a) are given directly below the program. Change the sampling frequency (for example, set $T_{\mathrm{s}}=0.001 \mathrm{~s}$ ) and repeat the previous process can immediately get the other results of Figure 2(b). Obviously, from the figure it is clearly 
shown the importance of sampling frequency in the discretization process of continuous-time signal: when satisfying the Nyquist sampling theorem (for example, sampling frequency equals to $5000 \mathrm{~Hz}$, which is greater than twice the highest frequency component of $2000 \mathrm{~Hz}$ ) as shown in Figure 2(a), the spectrum of sampled discrete-time sequence does not aliased, then the original continuous time signal can be recovered completely by passing a low-pass filter with an appropriate cutoff frequency and magnitude. When the sampling frequency $(1000 \mathrm{~Hz})$ is less than twice the highest frequency component as shown in Figure 2(b), the spectrum aliasing distortion of sampled discrete-time sequence occurs, and then the original continuous time signal is unable to recover. By modifying the sampling frequency, we can observe the corresponding changes of the spectrum of discrete time sequence. Here, it can be obviously felt the course teaching advantages with the help of Matlab Notebook: when teaching theoretical knowledge in the Mbook document, we can simulate the relevant knowledge in real-time as required, and do not need to switch to other application program window.

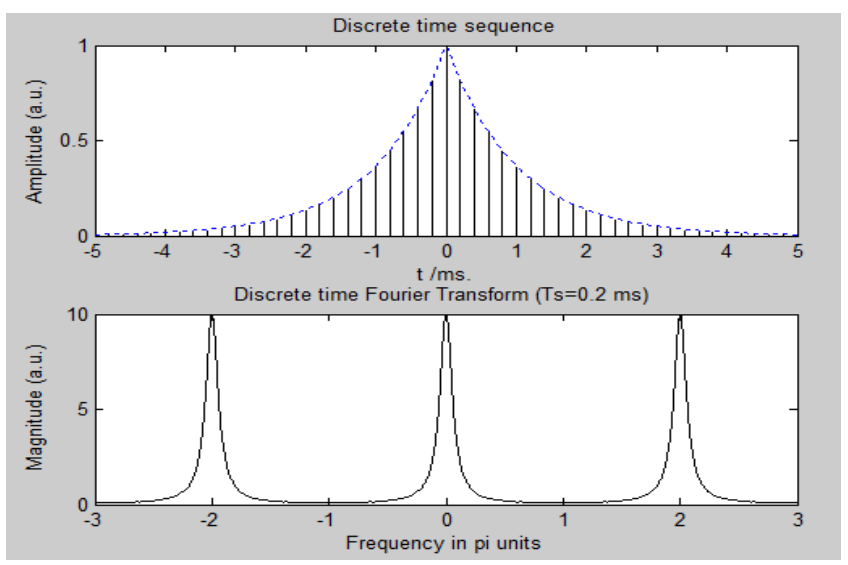

(a)

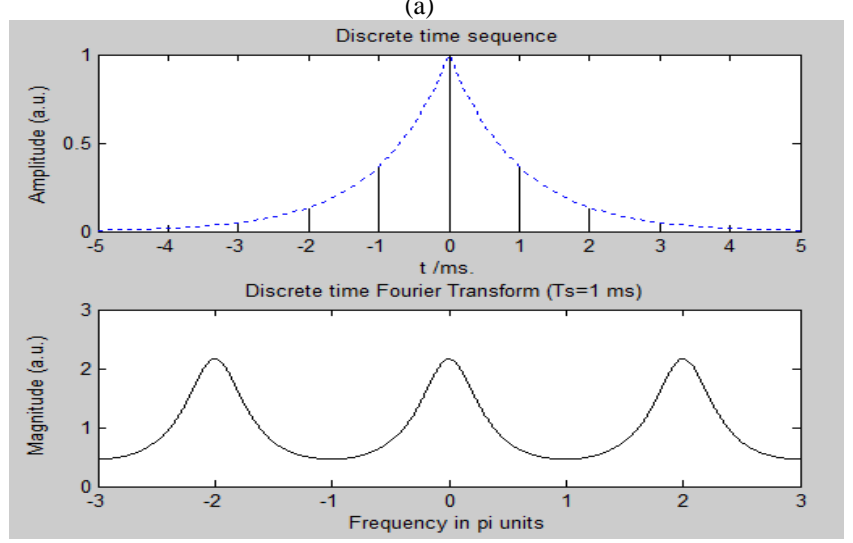

(b)

Figure 2. Discrete-time sequence obtained from sampling frequency of $5000 \mathrm{~Hz}(\mathrm{a})$ and $1000 \mathrm{~Hz}$ (b) and their corresponding discrete-time Fourier spectra

\section{CONCLUSION}

The Matlab Notebook achieves a seamless link of Matlab with Word software and makes full use of the both advantages, thus we can real-time program, calculate and display the results in the Word document. All these will be significance in the classroom teaching of Signal and System for the cases of needing to simulate the relevant knowledge points, or needing to calculate and give data and figure result again when modifying parameters. In fact, Matlab Notebook can be not only used for classroom teaching but also widely applied in various aspects such as writing technical reports, papers and monographs.

\section{ACKNOWLEDGMENT}

This work was financially supported by the excellent course construction of Signal and System of Ningbo University (JYJPKC201106)

\section{REFERENCES}

[1] G.Y. Jiang, G.Q. Hang, X.B. Zheng, "Signal and linear system (First Edition),” Beijing: Tsinghua University Press, 2012 (in Chinese)

[2] P. Q. Chen, "Digital signal processing tutorial (Third Edition)," Beijing: Tsinghua University Press, 2007 (in Chinese)

[3] P. Wallisch, M. Lusignan, M. Benayoun, T. I. Baker, A. S. Dickey, and N. G. Hatsopoulos, "MATLAB Tutorial," Matlab for Neuroscientists, 2009, pp. 7-56.

[4] S. Mitra, and T. Bose, "Processing of analytical signals using Matlab,” Chemometrics and Intelligent Laboratory Systems, vol. 22, 1994, pp. 3-16

[5] S. Bose, B. Friedlander, and A. Zeira, "MAT2DSP-A MATLAB tool for rapid feedback on the implementation requirements of signal processing algorithms,” Computer Standards \& Interfaces, vol. 20, 1999, pp. 474

[6] R. Gupta, J. N. Bera, and M. Mitra, "Development of an embedded system and MATLAB-based GUI for online acquisition and analysis of ECG signal,” Measurement, vol. 43, 2010, pp. 1119-1126

[7] S. K. Sen, and G. A. Shaykhian, "MatLab tutorial for scientific and engineering computations: International Federation of Nonlinear Analysts (IFNA)," Nonlinear Analysis: Theory, Methods \& Applications, vol. 71, 2009, pp. e1005-e1020 